\title{
Valores normales de las velocidades de flujo e índices de resistencia de las arterias oftálmica, central de la retina y ciliares cortas posteriores por ultrasonido Doppler color en población mexicana
}

\section{Normal values of flow velocity and resistance index of the ophthalmic, the central retinal, and the short posterior ciliary arteries by color Doppler ultrasound in Mexican population}

\author{
Álvaro D. Verdugo-Unigarro ${ }^{1 *}$, Marco A. Tobar-Marcillo ${ }^{2 *}$, Ernesto F. Martín-Biasotti ${ }^{1}$, Luis A. Padilla-Pére $\underline{\underline{z}}$ \\ y Jorge E. Ortiz-Gallegos ${ }^{1}$
}

${ }^{1}$ Servicio de Radiología e Imagen de Cabeza y Cuello, Instituto de Oftalmología Fundación Conde de Valenciana; ${ }^{2}$ Servicio de Medicina Interna, Hospital Regional Licenciado Adolfo López Mateos, Instituto de Seguridad y Servicios Sociales de los Trabajadores del Estado. Ciudad de Mếxico, México

\section{Resumen}

Antecedentes: El ultrasonido Doppler orbitario es útil para el diagnóstico de la enfermedad vascular orbitaria. Los valores en pacientes sanos varían dependiendo de la región geográfica; no se conocen los valores normales para la poblä́ción mexicana. Método: Estudio no experimental de tipo descriptivo transversal. Se incluyeron 154 pacientes mayores de èdad sin antecedentes patológicos ni quirúrgicos con repercusión oftalmológica, a quienes se realizó un ultrasonido Dopples de ambas órbitas para medir la velocidad sistólica máxima (VSM), la velocidad de fin de diástole y el índice de resistencia en las arterias oftálmica, central de la retina y ciliares cortas posteriores nasales y temporales. Resultados: La edad promedio de la población fue de 35,47 años y el 67,5\% eran mujeres. Sobre los valores de cada una de las mediciones realizadâs se aplicó la prueba de Kolmogórov-Smirnov para determinar la distribución paramétrica o no paramétrica de los resultadosicon sus respectivas medidas estadísticas. No se encontró variación de los valores relacionada con el sexo, y en cuantōa la edad se encontró una disminución de la VSM de la arteria oftálmica $(p=0.046)$ y de la VSM de las arterias ciliares cortas posteriores temporales $(p=0.048$ ) en los sujetos mayores de 50 años. Conclusiones: El ultrasonido Doppler es altamente accesible y reproducible, y resulta útil para obtener datos sobre el estado del flujo sanguíneo arterial orbitario. Puesto que hay diferencias importantes relacionadas con el país de procedencia, es imprescindible contar con nuestros propios datos en el estudio de patologías orbitarias.

Palabras clave: Arteria central de la retina. Arteria ciliar corta posterior nasal. Arteria ciliar corta posterior temporal. Afteria oftálmica. Ultrasonido Doppler.

Correspondencia:

*Marco A. Tobar Marcillo

Moras, 661

Col. del Valle, Del. Benito Juárez

C.P. 03100 , Ciudad de México, México

E-mail: marcotobar1@ @otmail.com Fecha de recepción: 29-03-2020

*Álvaro D. Verdugo-Unigarro

Fecha de recepción: 29-03-2020

Disponible en internet: 11-01-2021

E-mail: davemten@ hotmail.com

DOI: 10.24875/RMO.M20000141

Rev Mex Oftalmol. 2021;95(1):8-14

www.rmo.com.mx

0187-4519/@ 2020 Sociedad Mexicana de Oftalmología. Publicado por Permanyer. Este es un artículo open access bajo la licencia CC BY-NC-ND (http://creativecommons.org/licenses/by-nc-nd/4.0/). 


\section{Abstract}

Background: Orbital color Doppler ultrasound is a useful tool for the diagnosis of orbital vascular disease. The values in healthy patients vary depending on the geographic region; the normal values for the Mexican population are unknown. Methods: This is a non-experimental cross-sectional descriptive study; 154 patients of legal age without relevant ocular disorders or surgical history were included, who underwent a Doppler ultrasound of both orbits to measure the maximum systolic velocity (MSV), end-diastolic velocity and resistance index of the ophthalmic artery, the central retinal artery, the nasal short posterior ciliary artery and the temporal short posterior ciliary artery. Results: The mean age of the population was 35.47 years and $67.5 \%$ were women. The Kolmogórov-Smirnov test was used to determine the parametric or non-parametric distribution of the results with their respective statistical measures. There was no variation of the values regarding ${ }^{-}$ex; in terms of age, we found a decrease of the MSV of both the ophthalmic artery $(p=0.046)$ and the temporal short posterior ciliary artery $(p=0.048)$ in patients over 50 years. Conclusions: Doppler ultrasound is highly accessible and reproducible, and is useful for obtaining data on the state of orbital arterial blood flow. Since there are important differences by geographical region, it is essential to obtain our own data to study orbital disorders.

Key words: Central retinal artery. Nasal short posterior ciliary artery. Temporal short posterior ciliary artery. Ophthalmic artery. Doppler ultrasound.

\section{Introducción}

El ultrasonido Doppler (UD) es una modalidad de imagen que permite visualizar el flujo sanguíneo y sus características de manera dinámica en tiempo real. Esta técnica se describió en $1979^{1,2}$ y originalmente tuvo un amplio uso en investigaciones cardiológicas y del sistema vascular periférico, y en otros órganos sólidos como el riñón y el hígado ${ }^{1-4}$. La investigación de los trastornos de la órbita con UD se describió por primera vez en $1989^{5}$; desde entonces, esta técnica ha demostrado ser valiosa para la valoración de numerosas alteraciones orbitarias y oculares ${ }^{6}$. Sin embargo, existen pocos estudios que determinen correlaciones patológicas, por lo que recientemente hay mayor interés en la investigación en esta técnica de imagen.

El grupo más importante de trastornos en que el UD ha demostrado ser útil son aquellos que involucran alteraciones en la hemodinámica y la perfusión del ojo ${ }^{7}$, y resulta imprescindible, para mejorar los conceptos sobre la fisiopatología de los trastornos hipóxico-isquémicos del ojo y del nervio óptico, su estadificación y la evaluación del impacto de diversas modalidades de tratamiento de estas anomalías 3 .

El análisis de los vasos retrobulbares mediante UD evidencia que se desarrollan cambios hemodinámicos conforme avanza la edad de los pacientes, mostrando velocidades de flujo más bajas e índices de resistencia más altos los pacientes adultos mayores ${ }^{8}$, producto del envejecimiento de los vasos con remodelación en las capas íntima y media, lo que conduce a pérdida de elasticidad y rigidez arterial ${ }^{9,10}$. En estudios previos ya se han determinado los valores normales en pacientes sanos en otras partes del mundo, como Norteamérica ${ }^{11}$ y el Reino Unido ${ }^{12}$, y otros investigadores than encontrado que existen variaciones hemodinámicas de la circulación orbitaria valoradas mediante UD entre pacientes con diferentes orígenes étnicos, principalmente entre europeos y africanos, y han concluidoque las velocidades de flujos menores en los africanos pueden implicar un mecanismo que incrementê el riesgo de glaucoma ${ }^{13,14}$. Las velocidades de flujo sistólica y diastólica y los índices de resistencia enelos vasos retrobulbares dependen de múltiples factores, y por ello las distintas patologías que los afecten reflejaran variabilidad de los parámetros hemodinámicos vasculares determinados en la evaluación con UD ${ }^{15}$.

Recientemente se han publicado artículos que describen las alteraciones circulatorias orbitarias portuD en distintos estados patológicos primarios de la órbita, así como en algunas patologías sistémicas. Sin embargo, hay poca información en la literatura sobre las propiedades hemodinámicas de la circulación retrobulbar en población mexicana y latina en general,ppor lo que es relevante determinar la normalidad de sus parámetros circulatorios.

\section{Método}

\section{Población de estudio}

Se realizó un estudio descriptivo de tipo trasversal entre julio y noviembre de 2018 en el Instituto- de Oftalmología Fundación Conde de Valenciana, de la Ciudad de México, que incluyó 154 voluntarios saños, de ambos sexos y mayores de edad, elegidos de manera aleatoria y que fueron evaluados por un médico oftalmólogo. Se excluyeron aquellos con antecedentes 
de diabetes mellitus, hipotiroidismo, hipertiroidismo, hipertensión, infarto de miocardio o angina de pecho, infarto cerebral $o$ ataque isquémico transitorio, enfermedad o claudicación vascular periférica e hiperlipidemia, antecedente de cirugía cardiaca o vascular, antecedente de enfermedad ocular que no sea catarata o pterigión, en tratamiento con medicamentos sistémicos o tópicos oculares, y de otra nacionalidad diferente a la mexicana. Previamente a la realización del estudio se recabó el consentimiento informado individual a todos los pacientes. El estudio se realizó con autorización del comité de investigación institucional, con número de protocolo 2018.065 .

\section{Procedimiento}

Los pacientes estuvieron en reposo 15 minutos en sedestación antes de la realización del UD de la órbita. Se utilizó un equipo de ultrasonido Philiphs $\AA$ ClearVue 550 Release 3.0 con un transductor lineal multifrecuencia Active Array de 4 a $12 \mathrm{MHz}$. La duración de cada escaneo individual osciló entre 30 y 40 minutos, con el paciente en posición supina, y el transductor se aplicó suavemente sobre el párpado superior cerrado sin ejercer presión al acoplarlo con poca cantidad de gel conductivo.

Se realizaron exploraciones preliminares en modo $B$ en planos horizontales y verticales a través del ojo como procedimiento de detección. Se identificaron los vasos en Doppler color, se usó el volumen muestra de $0.5 \mathrm{~mm}$ y se ajustó el ángulo Doppler a los vasos antes de obtener el espectro. Para asegurar una información precisa de las velocidades, el ángulo Doppler no se ajustó a más de $60^{\circ}$, ya que con valores mayores las pequeñas variaciones en la asignación de la dirección de flujo se traducen en grandes variaciones en la estimación de la velocidad. Se registraron las formas de onda Doppler espectral y se recabó información de la arteria oftálmica (AO), la arteria central de la retina (ACR), la arteria ciliar corta posterior nasal (ACCPN) y la arteria ciliar corta posterior temporal (ACCPT), obteniendo la velocidad sistólica máxima (VSM), la velocidad de fin de diástole (VFD) y el índice de resistencia (IR).

\section{Análisis estadístico}

Se utilizó el programa SPSS Versión 25 para la tabulación de los datos y el análisis de los resultados. Se incluyeron variables cualitativas con aplicación de tablas de frecuencia y variables cuantitativas en las cuales se estableció la distribución por medio de la prueba de Kolmogórov-Smirnov. Cuando el valor de esta prueba fue menor de 0,07 se ajustó por Lillieforors. Para el caso de estas pruebas, la hipótesis nula corresponde a que las variables presentan distribución no paramétrica y la hipótesis alterna lo contrario. Parâ las variables con distribución paramétrica se estableciêron la media y la desviación estándar, y para las variablles con distribución no paramétrica, la mediana, los percentiles 5 y 95, y los límites superior e inferior:ios resultados de los flujos y de los índices de resistenicia en cada una de las arterias evaluadas se representaron en gráficos $Q-Q$.

\section{Resultados}

Se incluyeron 154 pacientes con exploración orbitaria bilateral, en total 308 exploraciones, y no hubo pérdida de datos. La edad tuvo una distribución no paramétrica, con una media de 35.48 años y cuna mediana de 32 años. El $67.5 \%$ (52) de los pacientes eran mujeres.

El estudio de UD se realizó en los cuatro territorios arteriales descritos (Fig. 1), cuyos resultados se-presentan en la tabla 1, clasificando en cada una de las arterias evaluadas la VSM y la VFD, y el IR. Para cōnocer la distribución de los valores en cada una dęlas variables se aplicó la prueba de Kolmogórov-Smirh̆ ov, y cuando su valor era $<0.07$ se ajustó por Lilliefors aplicación de las dos pruebas permitió rechazấ la hipótesis nula con una significancia del 95\% en ladedistribución de dos variables, la VSM de la ARC con una media de $12.134 \pm 2.942 \mathrm{~cm} / \mathrm{s}^{2}$ y el IR de la ACR con una media de $0.655 \pm 0.074$; el resto de las variables presentaron una distribución no paramétrica y se ăplicaron como métodos para la medición la mediana los límites superior e inferior, y los percentiles 5 y 95.

En la figura 2, mediante una gráfica $Q-Q$ se expresa la distribución de los valores observados y esperados para una distribución normal de la VSM, la VFD y ell $R$ en la AO. En la figura 3, mediante el mismo método de grafico $Q-Q$, se expresa la distribución de dichos valores en la ACR y se identifica la correlación entre valores observados y esperados para la VSM y eEIR, siendo estas variables las que mostraron distribuęión paramétrica. En las figuras 4 y 5 se muestra la distribución de las variables correspondiente a la ACCPN y la ACCPT, respectivamente.

En cuanto a la distribución por edad, 24 (15:6\%) pacientes tenían $\geq 50$ años. A través de una diferenicia de medias con prueba t se estableció que presentäron una VSM en la AO menor $(p=0.046)$ y una VSM en 

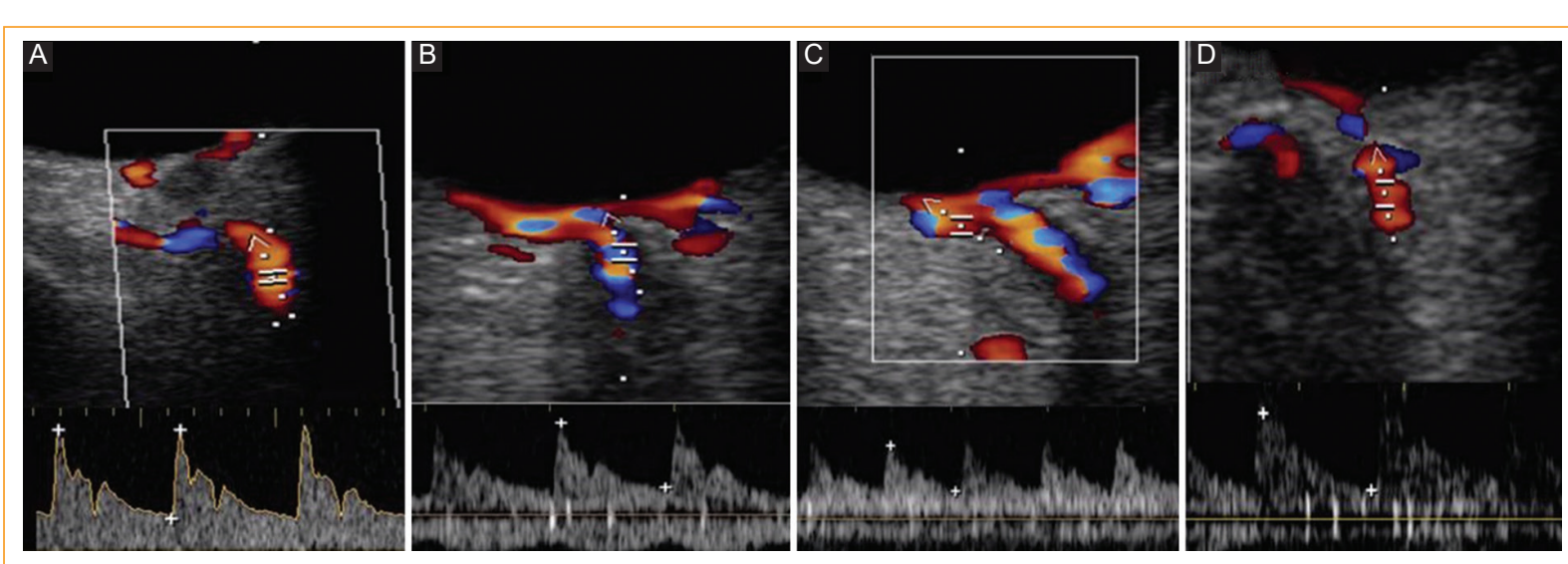

Figura 1. Imágenes en modo Doppler color y espectral de la arteria oftálmica $(A 0)(A)$, la arteria central de la retỉ̉a (ACR) (B), la arteria ciliar corta posterior nasal (ACCPN) (C) y la arteria ciliar corta posterior temporal (ACCPT) (D).

Tabla 1. Valores obtenidos por ultrasonido Doppler de la velocidad sistólica máxima (VSM), la velocidad de fin de diástole (VFD) y el índice de resistencia (IR) en la arteria oftálmica (AO), la arteria central de la retina (ACR), la arteria ciliar corta posterior nasal (ACCPN) y la arteria ciliar corta posterior temporal (ACCPT)

\begin{tabular}{|c|c|c|c|c|c|c|c|c|c|}
\hline & Media & Mediana & DE & P 5 & P 95 & $\begin{array}{l}\text { Límite } \\
\text { inferior }\end{array}$ & $\begin{array}{l}\text { Límite } \\
\text { superior }\end{array}$ & $\begin{array}{l}\text { Prueba } \\
\text { K-S }\end{array}$ & $\begin{array}{l}\text { Significaci } \\
\text { asintótic }\end{array}$ \\
\hline VSM en A0 $\left(\mathrm{cm} / \mathrm{s}^{2}\right), \mathrm{n}=308$ & 33.101 & 31.632 & 8.812 & 21.47 & 50.83 & 15.72 & 55.60 & 0.095 & $<0.001+$ \\
\hline VFD en $A 0\left(\mathrm{~cm} / \mathrm{s}^{2}\right), n=308$ & 8.824 & 8.267 & 3.271 & 4.52 & 13.77 & 3.12 & 19.90 & 0.082 & 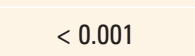 \\
\hline IR de $A 0, n=308$ & 0.734 & 0.741 & 0.067 & 0.626 & 0.837 & 0.55 & 0.86 & 0.052 & $0.046^{*}=$ \\
\hline VSM en ACR $\left(\mathrm{cm} / \mathrm{s}^{2}\right), \mathrm{n}=308$ & 12.138 & 11.952 & 2.942 & 7.27 & 16.57 & 5.26 & 21.70 & 0.041 & $0.200^{*}$ 을 \\
\hline VFD en ACR $\left(\mathrm{cm} / \mathrm{s}^{2}\right), n=308$ & 4.283 & 4.127 & 1.421 & 2.18 & 6.65 & 1.69 & 8.61 & 0.087 & $<0.001 \frac{\pi}{\sigma}$ \\
\hline IR de ACR, $\mathrm{n}=308$ & 0.655 & 0.661 & 0.074 & 0.522 & 0.769 & 0.39 & 0.80 & 0.047 & $0.094 * \frac{\overline{0}}{0}$ \\
\hline VSM en ACCPN $\left(\mathrm{cm} / \mathrm{s}^{2}\right), \mathrm{n}=308$ & 11.034 & 10.800 & 2.440 & 7.41 & 15.50 & 5.95 & 17.4 & 0.054 & $0.030^{*}$ \\
\hline VFD en ACCPN $\left(\mathrm{cm} / \mathrm{s}^{2}\right), \mathrm{n}=308$ & 4.627 & 4.283 & 1.454 & 2.59 & 7.51 & 2.02 & 8.31 & 0.094 & $<0.001$ \\
\hline IR de ACCPN, $n=308$ & 0.584 & 0.578 & 0.077 & 0.452 & 0.712 & 0.65 & 0.77 & 0.083 & $<0.001$ \\
\hline VSM en ACCPT $\left(\mathrm{cm} / \mathrm{s}^{2}\right), \mathrm{n}=308$ & 11.530 & 11.195 & 2.822 & 7.68 & 16.64 & 6.42 & 18.30 & 0.064 & $0.004 * \frac{\bar{\sigma}}{0}$ \\
\hline VFD en ACCPT $\left(\mathrm{cm} / \mathrm{s}^{2}\right), \mathrm{n}=308$ & 5.006 & 4.715 & 1.833 & 2.66 & 8.40 & 1.80 & 10.00 & 0.080 & $<0.001 \div$ \\
\hline IR de ACCPT, $n=308$ & 0.569 & 0.564 & 0.097 & 0.419 & 0.736 & 0.36 & 0.81 & 0.051 & $0.049^{*} \stackrel{\widetilde{\varrho}}{\Longleftarrow}$ \\
\hline \multicolumn{10}{|c|}{$\begin{array}{l}\text { DE: desviación estándar; K-S: Kolmogórov-Smirnov; P: percentil. } \\
\text { *Corrección de signifificación de Lilliefors. }\end{array}$} \\
\hline \multicolumn{4}{|c|}{$\begin{array}{l}\text { la ACCPT menor }(p=0.048) \text { en comparación con los } \\
\text { pacientes menores de } 50 \text { años. En el resto de las } \\
\text { mediciones no se alcanzó diferencia significativa, y de } \\
\text { igual manera en la distribución por sexo no hubo dife- } \\
\text { rencias significativas en los valores obtenidos. }\end{array}$} & \multicolumn{6}{|c|}{$\begin{array}{l}\text { patología orbitaria, ocular, del sistema nervioso central } \\
\text { y vascular sistémica. Para ello es importante contar con } \\
\text { valores normales de VSM, VFD e IR ajustados para la } \\
\text { población que se examina, puesto que existe evideñcia } \\
\text { que muestra cambios significativos en estos valöres } \\
\text { dependiendo del lugar de procedencia, como se mostró }\end{array}$} \\
\hline \multicolumn{4}{|l|}{ Discusión } & \multicolumn{6}{|c|}{$\begin{array}{l}\text { en un estudio que comparó a } 24 \text { pacientes de asčen- } \\
\text { dencia africana y } 34 \text { pacientes de ascendencia europpea }\end{array}$} \\
\hline \multicolumn{4}{|c|}{$\begin{array}{l}\text { El análisis de los flujos Doppler de los vasos retrobul- } \\
\text { bares mediante UD es una manera útil de determinar }\end{array}$} & \multicolumn{6}{|c|}{$\begin{array}{l}\text { indicando que estos últimos presentan valores de } \bar{Z} \text { F } \\
\text { e IR más altos, sin diferencias en la VSM en las cuatr }\end{array}$} \\
\hline
\end{tabular}




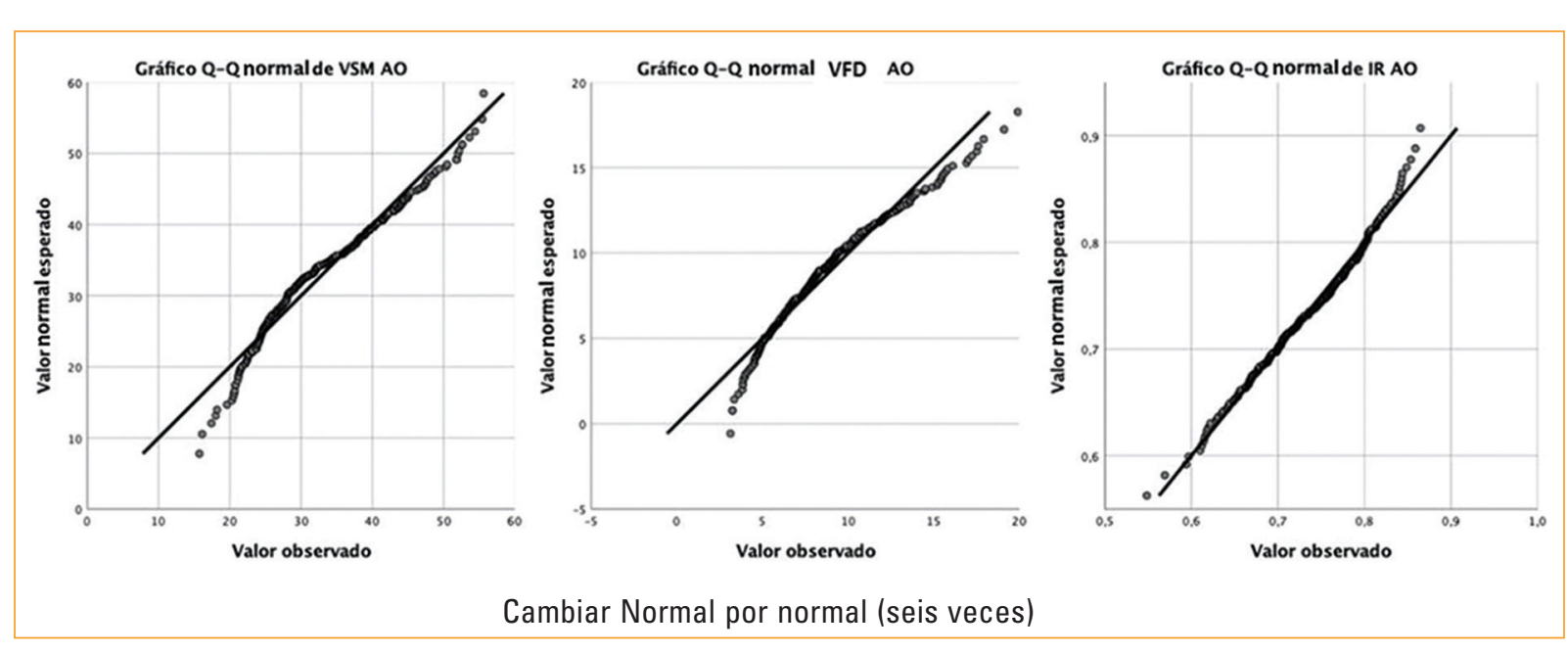

Figura 2. Gráfica 0-0 que muestra la distribución de los valores de velocidad sistólica máxima (VSM), velocidad de fin de diástole (VFD) e índice de resistencia (IR) en la arteria oftálmica (AO).

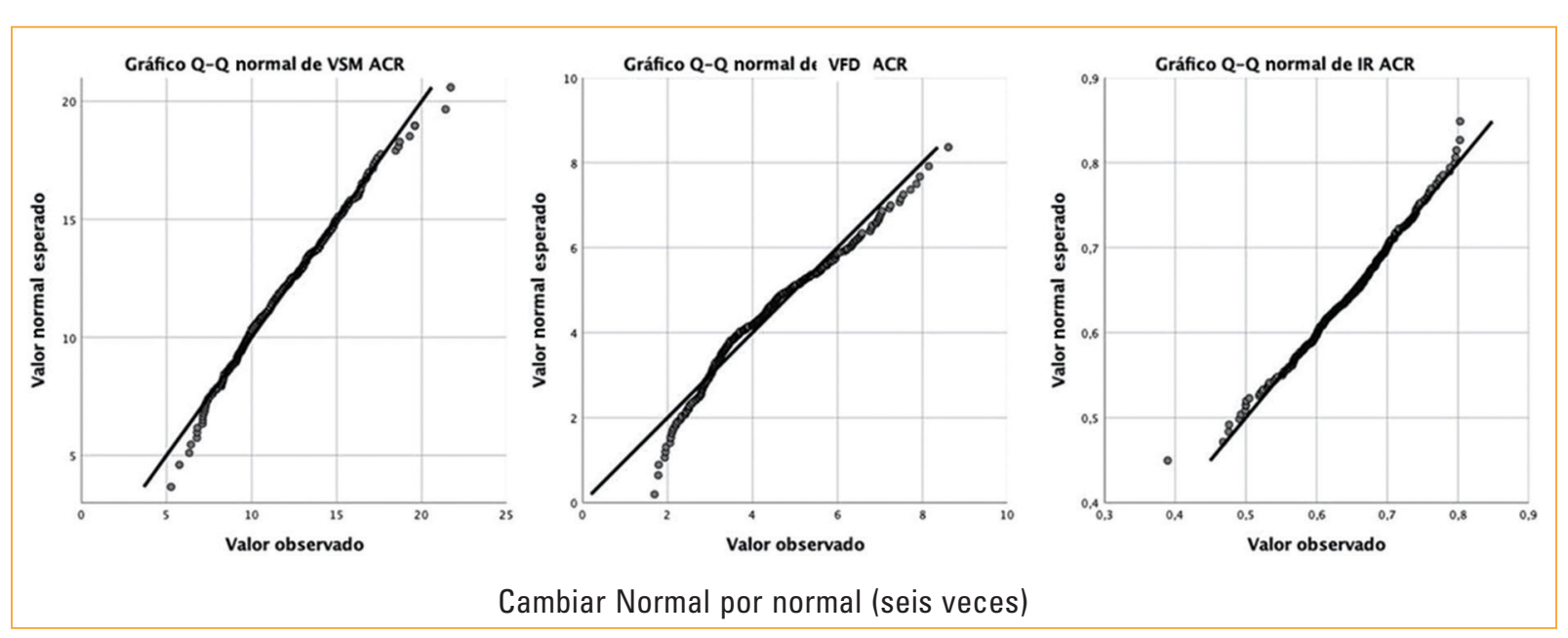

Figura 3. Gráfica 0-0 que muestra la distribución de los valores de velocidad sistólica máxima (VSM), velocidad đe fin de diástole (VFD) e índice de resistencia (IR) en la arteria central de la retina (ACR).
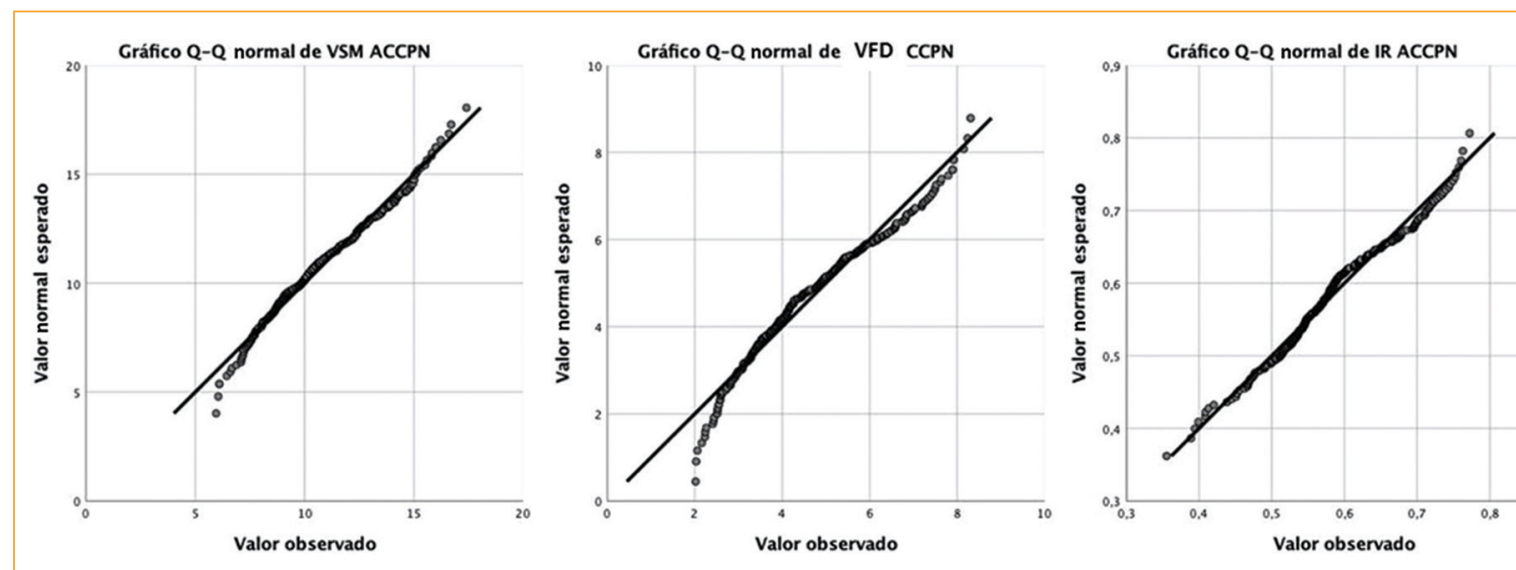

Cambiar Normal por normal (seis veces)

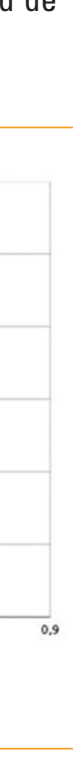

Figura 4. Gráfica Q-Q que muestra la distribución de los valores de velocidad sistólica máxima (VSM), velocidad đe fin de diástole (VFD) e índice de resistencia (IR) en la arteria ciliar corta posterior nasal (ACCPN). 


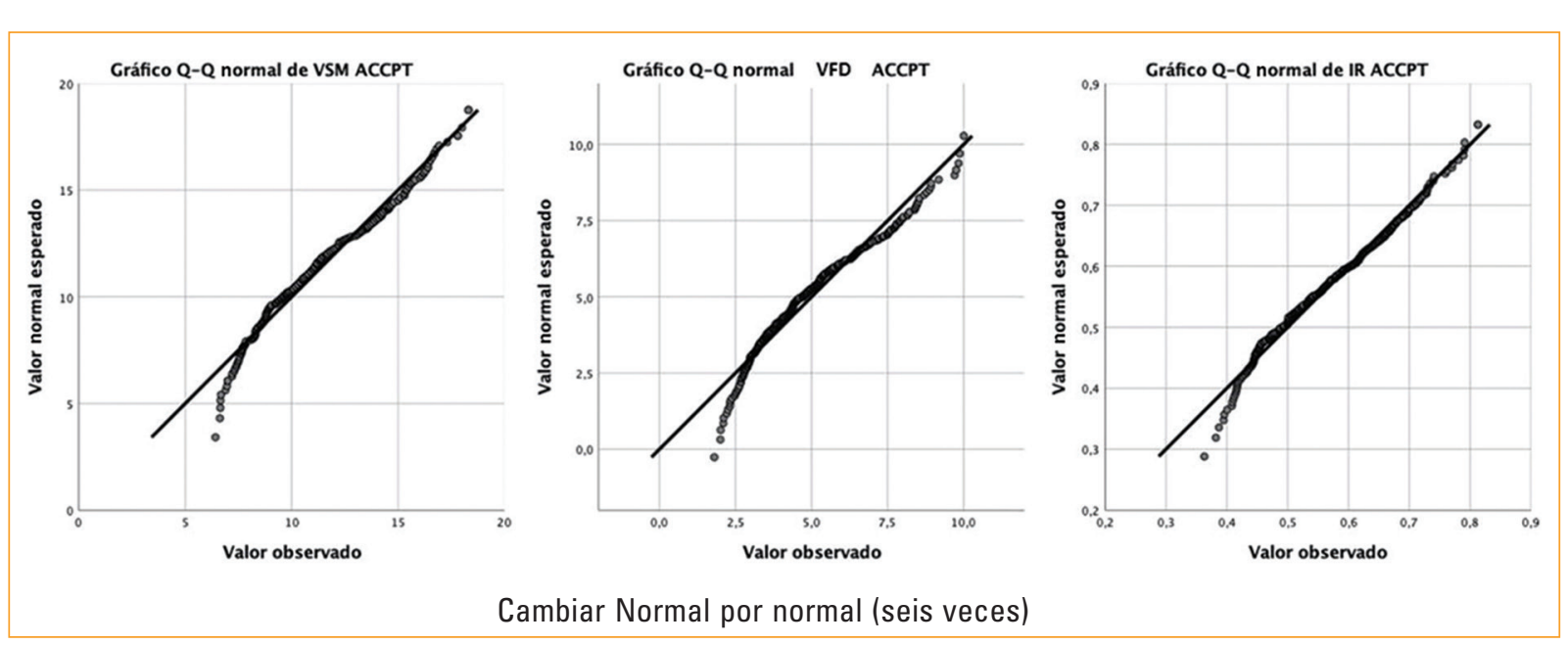

Figura 5. Gráfica 0-0 que muestra la distribución de los valores de velocidad sistólica máxima (VSM), velocidad de fin de diástole (VFD) e índice de resistencia (IR) en la arteria ciliar corta posterior temporal (ACCPT).

arterias examinadas ${ }^{13,14}$. Al comparar estos valores con los de nuestra muestra observamos que los pacientes mexicanos presentan velocidades de flujo más altas, con menor IR. Sin embargo, al compararlos con otros registros de pacientes sanos se encuentran variaciones importantes: un estudio en población canadiense ${ }^{11}$ que valoró los flujos en la $\mathrm{AO}$ de 60 pacientes mostró una VSM de $45,1 \pm 7,8 \mathrm{~cm} / \mathrm{s}^{2}$ y una VFD de $12,0 \pm 3,4 \mathrm{~cm} /$ $\mathrm{s}^{2}$, mayores que las encontradas en nuestros pacientes, y otro estudio desarrollado en el Reino Unido ${ }^{12}$ con 80 pacientes sanos midió las velocidades de flujo y los IR, en las cuatro arterias exploradas en nuestro estudio, $y$ halló de igual manera una VSM y una VFD en la AO significativamente mayores, sin variación en las velocidades en la ACR, la ACCPN y la ACCPT.

Existe variación de algunos valores respecto a la edad de los pacientes. En el trabajo de Modrzejewska, et al. ${ }^{8}$ se incluyeron 162 pacientes sanos que se dividieron en cinco grupos según la edad, y en el grupo de pacientes de 68-80 años se observaron una VSM y una VDM reducidas en la ACR, así como IR aumentados en la AO, la $A C R$, la ACCPN y la ACCPT. Estas diferencias están en relación con los cambios vasculares propios de la senectud, consistentes en esclerosis de las paredes arteriales. En nuestro análisis, que involucró un mayor número de pacientes, únicamente encontramos una reducción significativa de la VSM en la AO y en la ACCPT.

En cuanto a la distribución por sexo, un estudio que involucró 128 pacientes encontró una VDM reducida en mujeres de mayor edad ${ }^{16}$. En nuestros pacientes no se halló ninguna diferencia significativa en la medición según el sexo, incluso ajustando por la edad.
Encontrar estas diferencias entre nuestros resulltados y los reportados en la literatura internacional'permite establecer parámetros de normalidad en población latina, específicamente en población mexicana.

Teniendo esta herramienta podemos realizar un mejor estudio de patologías prevalentes, como la.örbitopatía tiroidea, que ha demostrado cambios hemodinámicos de la vena oftálmica superior como marcådor de estasis venosa identificados en esta etiología pesar de que el flujo arterial orbitario ha sido menos estudiado, es un marcador de lesión endotelial quę se manifiesta en un aumento de las velocidades de flujo $y$ de los $I^{18}$. También se ha encontrado que los pacientes diabéticos, en especial los más afectados por retinopatía diabética, tienen menores VSM y $\overline{\forall D F}$ en la ACR, con mayor IR tanto en la ACR como en la $\mathrm{AO}^{19}$. Algunos autores sostienen que una de las gausas del glaucoma es el origen vascular, hecho que ha tomado importancia en los últimos años, y la evaluación mediante UD ha demostrado ser una herramiênta muy útil. Los hallazgos ecográficos que fundameñtan esta teoría son la disminución de la VFD en la AGR y el aumento del IR de la ACCPN y de la ACCPT en la fase aguda de la enfermedad, mientras que en la fase crónica se identifica un descenso marcado de la VSM y de la VFD en estos últimos vasos, cambios que se asocian con progresión de la enfermedad ${ }^{20-22}$.

\section{Conclusión}

EI UD en un herramienta accesible, reproducible y de bajo costo, útil para obtener datos sobre las 
velocidades de flujo y los IR en las arterias orbitarias. El presente estudio corrobora los hallazgos presentados en otras series, como la disminución de la VSM y de la VFD, y el aumento del IR a medida que la edad de los pacientes progresa. De la misma manera, encontramos que en población mexicana existen valores menores de la VSM y la VFD, en comparación con otras poblaciones, sin encontrar un factor que determine estos cambios.

Por lo anterior, era de gran importancia, para el estudio de patologías oftalmológicas prevalentes en nuestro medio, contar con valores normales para población mexicana que nos permitan hacer una mejor evaluación y diagnóstico. Se requieren más estudios que involucren pacientes de otras partes de México y de otros países de América Latina.

\section{Conflicto de intereses}

Los autores declaran no tener ningún conflicto de intereses.

\section{Responsabilidades éticas}

Protección de personas y animales. Los autores declaran que los procedimientos seguidos se conformaron a las normas éticas del comité de experimentación humana responsable y de acuerdo con la Asociación Médica Mundial y la Declaración de Helsinki.

Confidencialidad de los datos. Los autores declaran que han seguido los protocolos de su centro de trabajo sobre la publicación de datos de pacientes.

Derecho a la privacidad y consentimiento informado. Los autores han obtenido el consentimiento informado de los pacientes y/o sujetos referidos en el artículo. Este documento obra en poder del autor de correspondencia.

\section{Bibliografía}

1. Mitchell DG. Color Doppler imaging: principles, limitations, and artifacts. Radiology. 1990;177:1-10.

2. Aburn NS, Sergott RC. Orbital colour Doppler imaging. Eye. 1993;7:639-47.

3. Foley WD. Vascular ultrasound: a radiologist perspective. Echocardiography. 1992;9:537-45.

4. McNaughton DA, Abu-Yousef MM. Doppler US of the liver made simple. RadioGraphics. 2011;31:161-88.

5. Erickson SJ, Hendrix LE, Massaro BM, Harris GJ, Lewandowski MF, Foley WD, et al. Color Doppler flow imaging of the normal and abnormal orbit. Radiology. 1989;173:511-6.

6. Guthoff RF, Berger RW, Winkler P, Helmke K, Chumbley LC. Döppler ultrasonography of the ophthalmic and central retinal vessels. ArchOphthalmol. 1991;109:532-6.

7. Stalmans I, Vandewalle E, Anderson DR, Costa VP, Frenkel RE, Garhofer $\mathrm{G}$, et al. Use of colour Doppler imaging in ocular blood flow research. Acta Ophthalmol. 2011;89:e609-30.

8. Modrzejewska M, Siesky B, Amireskandari A, Holland S, Grzesiak W, Zaborski $D$, et al. Parameters characterizing age-dependent retrobulbar circulation in healthy subjects measured by color Doppler ultrasonography. Curr Eye Res. 2015;40:729-36.

9. Bolton E, Rajkumar C. The ageing cardiovascular system. ReV Clin Gerontol. 2011;21:99-109.

10. O'Rourke MF, Hashimoto J. Mechanical factors in arterial aging: a êtinical perspective. J Am Coll Cardiol. 2007;50:1-13.

11. Rojanapongpun $P$, Drance SM. Velocity of ophthalmic arterial flow ded by Doppler ultrasound in normal subjects. Am J Ophthaimol. 1993;115:174-80.

12. Baxter GM, Williamson TH. Color Doppler imaging of the eye: normâa ranges, reproducibility, and observer variation. J Ultrasound Med. 1995;14:91-6.

13. Kaskan B, Ramezani K, Harris A, Siesky B, Olinde C, WuDunn Dicet al. Differences in ocular blood flow between people of African and European descent with healthy eyes. J Glaucoma. 2016;25:709-15.

14. Siesky B, Harris A, Racette L, Abassi R, Chandrasekhar K, Tobe LA, et al. Differences in ocular blood flow in glaucoma between patients of African and European descent. J Glaucoma. 2015;24:117-21.

15. Erickson SJ, Hendrix LE, Massaro BM, Harris GJ, Lewandowski MF, Foley WD, et al. Color Doppler flow imaging of the normal and abnormal orbit. Radiology. 1989;173:511-6.

16. Harris A, Harris M, Biller J, Garzozi H, Zarfty D, Ciulla TA, et al. Äging affects the retrobulbar circulation differently in women and men Arch Ophthalmol. 2000;118:1076-80.

17. Monteiro ML, Angotti-Neto HA, Benabou JE, Betinjane AJ. Color Dopppler imaging of the superior ophthalmic vein in different clinical forms of Graves' orbitopathy. Jpn J Ophthalmol. 2008;52:483-8.

18. Gonçalves AC, Gebrim EM, Monteiro ML. Imaging studies for diagn̊osing Graves' orbitopathy and dysthyroid optic neuropathy. Clinics (Sao Paulo). 2012;67:1327-34.

19. Meng N, Liu J, Zhang Y, Ma J, Li H, Qu Y. Color Doppler imaging ânalysis of retrobulbar blood flow velocities in diabetic patients without oP with retinopathy: a meta-analysis. J Ultrasound Med. 2014;33:1381-9:-

20. Gherghel D, Orgül S, Gugleta K, Gekkieva M, Flammer J. Relationship between ocular perfusion pressure and retrobulbar blood flow in patients with glaucoma with progressive damage. Am J Ophthalmol. 2000;130:597-605.

21. Ehrlich R, Harris A, Siesky BA, Moss AM, Ramanathan M, Pickete MA, et al. Repeatability of retrobulbar blood flow velocity measured $\bar{y}$ sing color Doppler imaging in the Indianapolis Glaucoma Progression Study. J Glaucoma. 2011;20:540-7.

22. Srikanth K, Kumar MA, Selvasundari S, Prakash ML. Colour Dōppler imaging of ophthalmic artery and central retinal artery in glaucoma patients with and without diabetes mellitus. J Clin Diagn Res. 2014;8:VC01-2 